\title{
The correlation of WDR76 expression with survival outcomes and immune infiltrates in lung adenocarcinoma
}

\author{
Likui Fang ${ }^{1}$, Guocan Yu ${ }^{1}$, Wenfeng Yu ${ }^{1}$, Gang Chen ${ }^{1}$, Bo Ye ${ }^{\text {Corresp. } 1}$ \\ ${ }^{1}$ Department of Thoracic Surgery, Affiliated Hangzhou Chest Hospital, Zhejiang University School of Medicine, Hangzhou, China \\ Corresponding Author: Bo Ye \\ Email address: B1618142@zju.edu.cn
}

Background: WD repeat domain 76 (WDR76) is a predicted member of the WD40-repeat-containing domain superfamily and possibly involves in various biological processes, but its function in cancers is poorly characterized. This study aimed to evaluate the role of WDR76 in the prognosis and immune infiltrates of lung adenocarcinoma (LUAD).

Methods: WDR76 expressions in LUAD tissues and normal tissues were primarily compared by The Cancer Genome Atlas (TCGA) database, and were validated in cohorts from Gene Expression Omnibus (GEO) database. The associations between WDR76 expression and clinicopathologic characteristics were analyzed. Kaplan-Meier and Cox regression analyses were performed to determine the impact of WDR76 expression on survival outcomes. The protein interaction network of WDR76 was built using STRING website. TIMER and GEPIA databases were used to investigate the correlation between WDR76 expression and immune infiltrates.

Results: WDR76 expression was elevated in LUAD $(P<0.001)$ and high WDR76 expression was associated with advanced $\mathrm{N}$ stage, $\mathrm{M}$ stage and pathologic stage. Expectedly, high WDR76 expression significantly correlated with poor survival outcomes and was the independent risk factor for overall survival (OS) (HR 1.468, 95\% Cl 1.031-2.089, $\mathrm{P}=0.033$ ) and disease specific survival (DSS) (HR 1.764, $95 \% \mathrm{Cl} 1.095-2.842, \mathrm{P}=0.020)$. DDB1 and LSH were the important proteins interacting with WDR76. WDR76 expression correlated with CD8+ T cells presence and was also positively associated with levels of inhibitory receptors.

Conclusion: WDR76 expression was involved in the regulation of immune infiltrates and had predictive value for prognosis in LUAD. 
1 The correlation of WDR76 expression with survival outcomes and immune infiltrates in lung

2 adenocarcinoma

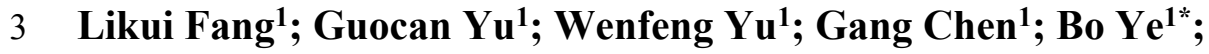

$4{ }^{1}$ Department of Thoracic Surgery, Affiliated Hangzhou Chest Hospital, Zhejiang University

5 School of Medicine, Hangzhou 310003, China.

6

7 Running title: WDR76 expression in lung adenocarcinoma

9 *Correspondence: Dr. Bo Ye, Department of Thoracic Surgery, Affiliated Hangzhou Chest

10 Hospital, Zhejiang University School of Medicine, Hangzhou 310003, China

E-mail: B1618142@zju.edu.cn 


\section{Abstract}

Background: WD repeat domain 76 (WDR76) is a predicted member of the WD40-repeat-

containing domain superfamily and possibly involves in various biological processes, but its

function in cancers is poorly characterized. This study aimed to evaluate the role of WDR76 in the

prognosis and immune infiltrates of lung adenocarcinoma (LUAD).

Methods: WDR76 expressions in LUAD tissues and normal tissues were primarily compared by

The Cancer Genome Atlas (TCGA) database, and were validated in cohorts from Gene Expression

Omnibus (GEO) database. The associations between WDR76 expression and clinicopathologic

characteristics were analyzed. Kaplan-Meier and Cox regression analyses were performed to

determine the impact of WDR76 expression on survival outcomes. The protein interaction network

of WDR76 was built using STRING website. TIMER and GEPIA databases were used to

investigate the correlation between WDR76 expression and immune infiltrates.

Results: WDR76 expression was elevated in LUAD $(\mathrm{P}<0.001)$ and high WDR76 expression was

associated with advanced $\mathrm{N}$ stage, $\mathrm{M}$ stage and pathologic stage. Expectedly, high WDR76

expression significantly correlated with poor survival outcomes and was the independent risk

factor for overall survival (OS) $(\mathrm{HR} 1.468,95 \% \mathrm{CI} 1.031-2.089, \mathrm{P}=0.033)$ and disease specific

survival (DSS) (HR 1.764, 95\%CI 1.095-2.842, $\mathrm{P}=0.020)$. DDB1 and LSH were the important

proteins interacting with WDR76. WDR76 expression correlated with CD8+ T cells presence and was also positively associated with levels of inhibitory receptors.

42 Conclusion: WDR76 expression was involved in the regulation of immune infiltrates and had 
predictive value for prognosis in LUAD.

Keywords: lung adenocarcinoma, WDR76, prognosis, immune infiltrates, TCGA

\section{Introduction}

Lung cancer is one of the most common malignant tumors and the leading cause of cancer-related mortality worldwide[1, 2]. Non-small cell lung cancer (NSCLC) accounts for more than $80 \%$ cases of lung cancer and lung adenocarcinoma (LUAD) is the most frequent histological subtype[3]. Most cases of LUAD were diagnosed at advanced or metastatic stage and systematic therapy should be advised[4]. Chemotherapy is not always an effective approach, whereas tyrosine kinase inhibitors (TKIs) therapy has shown a good activity in oncogene driven disease[5, 6]. In the era of immunotherapy, there are increasing evidences supporting the use of immune checkpoint inhibitors (ICIs) in the systematic therapy[7]. Despite the encouraging data, there are still some problems such as limited patient response rates and drug resistance. Ferroptosis is a form of regulated cell death that mainly results from iron accumulation and lipid peroxidation[8]. Ferroptosis could affect the efficacy of chemotherapy, radiotherapy and immunotherapy, and targeting ferroptosis signaling could improve the outcomes from those therapies $[9,10]$. Therefore, inducing ferroptosis is becoming a promising strategy in the treatment of NSCLC $[11,12]$.

WD repeat domain 76 (WDR76) is a predicted member of the WD40-repeat-containing domain superfamily, whose function is poorly characterized in cancers[13]. A recent research indicated that WDR76 could activate lipid metabolism-associated genes to inhibit ferroptosis in dependent 
64

manner of lymphoid-specific helicase (LSH) which acted as an oncogene in lung cancer[14]. However, researches focusing on WDR76 are limited, and its role in immune microenvironment and prognosis of LUAD is unclear. This study explored the prognostic significance of WDR76 expression in LUAD through bioinformatics analysis of the clinical characteristics from The Cancer Genome Atlas (TCGA). TIMER and GEPIA databases were used to investigate the associations of WDR76 expression with infiltrated immune cells and their corresponding gene marker sets. Our results demonstrated that high expression of WDR76 was associated with poor prognosis in LUAD, and WDR76 expression positively correlated with not only CD8+ T cell presence but also inhibitory receptors such as PDCD1, LAG3 and TIM-3. Thus, it was plausible that WDR76 could inhibit ferroptosis and reduce $\mathrm{T}$ cell cytotoxicity to promote LUAD progression.

\section{Methods}

Data source

The data of LUAD patients with the expression of mRNA and matching clinicopathological information were obtained from The Cancer Genome Atlas (TCGA) database (https://portal.gdc.cancer.gov/). The database is publicly open-access and the approval of local ethics committee is not necessary. A total of 535 patients with LUAD and their clinicopathologic characteristics were included in this study. The gene expression profiling data sets (GSE140797, GSE27262 and GSE18842) were obtained from Gene Expression Omnibus (GEO) database (https://www.ncbi.nlm.nih.gov/gds) to verify the differential expression of WDR76 between tumor 
tissues and normal tissues.

\section{TIMER and GEPIA database analysis}

The Tumor Immune Estimation Resource (TIMER) is a public website (http://timer.cistrome.org/) which is a comprehensive resource for systematic analysis of immune infiltrates across 32 cancer types. The correlation of WDR76 expression with the abundance of six types of infiltrating immune cells (CD8+ T cells, CD4+ T cells, macrophages, B cells, dendritic cells and neutrophils) in LUAD was evaluated by TIMER database[15].

The Gene Expression Profiling Interactive Analysis (GEPIA) is an online database which provides key interactive and customizable functions including differential expression analysis, correlation analysis, survival analysis, similar gene detection and dimensionality reduction analysis[16]. The associations of WDR76 expression with multiple markers for immune cells were investigated by GEPIA database.

\section{Protein-protein interaction comprehensive analysis}

The Search Tool for the Retrieval of Interacting Genes/Proteins (STRING) is a website (https://string-db.org/) hosting a great collection of integrated and consolidated protein-protein interaction data[17]. We obtained the protein-protein interaction (PPI) network information of WDR76 by the online tool STRING. The confidence score $>0.7$ was considered significant. 
106

107 The patients were divided into two groups according to the median expression of WDR76. The 108

109

110

111

112

113

114

115

116

117

118

119

120

121

122

123

124

125

126

Statistical analysis

measurement data was statistically analyzed with $t$ test or Mann-Whitney $U$ test according to the data distribution. As for the numeration data, chi-square test or corrected chi-square test or the Fisher's exact test was used, depending on the actual situation. Multiple testing correction was performed by Bonferroni method when testing WDR76 and immune cell markers in GEPIA. Kaplan-Meier curve and log-rank test were performed to analyze the survival impact of WDR76 expression. Survival outcomes included overall survival (OS), disease specific survival (DSS) and progress free survival (PFS). Cox regression analysis was performed to verify the effect of WDR76 expression on survival outcomes. All the above analysis was conducted by R statistical software (version 3.6.3) and SPSS software (version 24.0). Statistical significance was set at $\mathrm{P}$ value $<0.05$ (All P values presented were 2-sided).

\section{8}

\section{Results}

\section{Baseline characteristics}

A total of 535 LUAD samples and 59 normal tissue samples from the TCGA database were incorporated in the study. The baseline characteristics of patients including age, gender, smoking history and pathological stage were summarized in Table 1.

\section{High WDR76 expression in LUAD}

The gene expression level of WDR76 was significantly higher in tumor samples than that in normal 
127 tissue samples in TCGA database $(\mathrm{P}<0.001$, Figure $1 \mathrm{~A})$. In the analysis of associations between

128 WDR76 expression and clinicopathologic characteristics in LUAD (Figure 1B-H), the results

129 showed that no significant association of WDR76 expression with age $(\mathrm{P}=0.156)$ and smoking

130 history $(\mathrm{P}=0.277)$. Higher expression of WDR76 was observed in male $(\mathrm{P}=0.012)$, advanced $\mathrm{N}$

131 stage $(\mathrm{P}=0.017)$ and $\mathrm{M}$ stage (0.007). Comparing with T1 stage, WDR76 expression significantly

132 increased in $\mathrm{T} 2$ stage $(\mathrm{P}=0.008)$, but the difference was not observed in $\mathrm{T} 3$ and $\mathrm{T} 4$ stage. In

133 addition, WDR76 expression also increased in advanced pathologic stage (Stage III and IV) in

134 comparison to early stage (Stage I and II) $(\mathrm{P}=0.003)$.

135

136 In order to verify the differential expression, we further compared WDR76 expression between

137 LUAD and normal tissues in data sets GSE140797 (7 pairs tumor and normal tissues), GSE27262

138 (25 pairs tumor and normal tissues) and GSE18842 (44 pairs tumor and normal tissues) from GEO

database (Figure 2A-C). The results showed increased expression of WDR76 in LUAD compared

to normal tissues $(\mathrm{P}=0.009,0.020$ and $<0.001$, respectively).

High WDR76 expression showing poor survival outcomes

143 Kaplan-Meier survival analysis showed that the patients with high expression of WDR76

144 correlated with inferior overall survival (OS) $(\mathrm{P}=0.004)$, disease specific survival (DSS) $(\mathrm{P}=0.002)$

and progress free survival (PFS) $(\mathrm{P}=0.003)$, as shown in Figure 3A-C. In the univariate Cox model,

both high WDR76 expression and advanced pathologic stage were a negative predictor for OS,

DSS and PFS. Gender, age and smoking history were not associated with survival outcomes. 
148 Multivariate Cox regression analysis further proved that WDR76 expression was the independent

149 risk factor for OS (hazard ratio (HR) 1.468, 95\% confidence interval (CI) 1.031-2.089, $\mathrm{P}=0.033$ )

150 and DSS (HR 1.764, 95\%CI 1.095-2.842, $\mathrm{P}=0.020$ ) (Figure 4).

151

152

Constructing protein interaction networks

153

STRING tool was used to analyze the PPI network of WDR76 protein to determine their interactions. The top 10 proteins and corresponding gene symbols, annotations and scores were

listed in Figure 5. WDR76 protein most significantly interacted with DNA damage-binding protein

1 (DDB1), followed by lymphoid-specific helicase (HELLS, LSH), thymocyte nuclear protein

1(THYN1) and DNA repair protein complementing XP-C cells (XPC).

Association between WDR76 expression and immune infiltrates

We explored the association between WDR76 expression and six kinds of infiltrating immune cells (CD8+ T cells, macrophages, neutrophils, B cells, CD4+ T cells and dendritic cells) in LUAD

from the TIMER database (Figure 6). The results showed significant positive associations of

WDR76 expression with CD8+ T cells $(\mathrm{R}=0.269, \mathrm{P}=1.34 \mathrm{e}-09)$, macrophages $(\mathrm{R}=0.221, \mathrm{P}=7.39 \mathrm{e}-$

07) and neutrophils $(\mathrm{R}=0.353, \mathrm{P}=6.50 \mathrm{e}-16)$, and negative associations with $\mathrm{B}$ cells $(\mathrm{R}=-0.22$,

$\mathrm{P}=8.15 \mathrm{e}-07)$ and $\mathrm{CD} 4+\mathrm{T}$ cells $(\mathrm{R}=-0.096, \mathrm{P}=3.34 \mathrm{e}-02)$, and no association with tumor purity and dendritic cells.

To further explore the possible role of WDR76 expression in immune infiltrates, GEPIA database 
169

170

171

172

173

174

175

176

was used to assess relationships between WDR76 and immune marker sets of various immune cells. The results showed that WDR76 expression was statistically associated with the levels of some immune sets (Table 2). It should be noted that inhibitory receptors such as PDCD1, LAG3 and Tim-3 were significantly and positively associated with WDR76 expression.

\section{Discussion}

WDR76 is a nuclear WD40 protein, which possibly involves in a variety of distinct biological processes including DNA damage repair, cell cycle progression, apoptosis, gene expression regulation and protein quality control[13, 18]. Studies aimed at unveiling novel DNA methylation readers have shown that WDR76 specifically binds 5-hydroxymethylcytosine $(5 \mathrm{hmC})$ and it acts as a specific reader of $5 \mathrm{hmC}$, hence suggesting involvement of WDR76 in epigenetic transcriptional regulation[19]. Despite the possible involvement of WDR76 in multiple biological processes, its exact role in cancers remains to be elucidated.

Therefore, we conducted the study to explore the effect of WDR76 expression on the progression and prognosis of LUAD on the basis of various databases including TCGA, GEO, TIMER and GEPIA. The results showed that WDR76 expression significantly increased in LUAD in comparison to normal tissues. Similarly, high WDR76 expression was observed in advanced N stage, $\mathrm{M}$ stage and pathologic stage, while the effect was not obvious in T stage, which might be caused by the relatively small samples of T3 and T4 stage (49 and 19, respectively). Interestingly, WDR76 expression was higher in male than in female without different expression in smoking 
190

191

192

193

194

195

196

197

198

199

200

201

202

203

204

205

206

207

208

209

210

history, whose mechanism needed to be further studied. In addition, Kaplan-Meier survival analysis revealed that low expression of WDR76 in LUAD had favorable OS, DSS and PFS compared with high expression. WDR76 expression was further proven to be an independent predictive factor of prognosis by Cox regression analysis. These links to LUAD have been supported by the evidence of other databases. The OncoLnc database (http://www.oncolnc.org/) shows correlation of aberrant WDR76 expression with shorter survival in LUAD. Furthermore, the Human Protein Atlas lists WDR76 as an unfavorable prognostic marker in various cancers including lung cancer[20].

Given the vital role of WDR76 expression in LUAD, determining the protein interaction network of WDR76 was of high importance. DDB1 and LSH were identified as the important proteins which interacted with WDR76 based on the analysis from STING website. DDB1 is required for DNA repair and has been reported to involve in conferring tolerance to DNA replication stress to promote LUAD development[21]. LSH, a member of the ATP-dependent helicase in sucrose nonfermenting 2 (SNF2), plays an important role in inhibiting ferroptosis and promoting lung tumorigenesis[22]. A recent study showed that LSH could contribute the recruitment of WDR76 to the metabolic gene promoters such as stearoyl-CoA desaturase 1(SCD1) and fatty acid desaturases 2 (FADS2) to upregulate their expressions, which could affect the intracellular levels of iron and lipid reactive oxygen species (ROS) to block ferroptosis[14]. WDR76 was also identified as a molecular inhibitor of the Cullin-4 RING ubiquitin ligase (CRL4) system for stability control of LSH and as a crucial regulator in epigenetic regulation of ferroptosis[23]. 
212 In recent years, immunotherapy with immune checkpoint inhibitors (ICIs) has revolutionized the

213 clinical management of patients with NSCLC, but only a minority of patients experience durable

214 response[24]. A recent report indicated that immunotherapy-activated CD8+ T cells could enhance

215 specific lipid peroxidation in tumor cells to induce ferroptosis, and increased ferroptosis could

216 contribute to the antitumor efficacy of immunotherapy[25]. However, the interaction between

217 ferroptosis and immune system is complicated, and does not just lead to a completely positive or

218 negative effect[10]. In this study, we tried to reveal whether WDR76 played an important role in

219 immune infiltrates by performing bioinformatics analysis of public data. In the TIMER database,

220 WDR76 expression showed negative correlation with B cells and CD4+ T cells. Infiltrating B cells

221 and CD4+ T cells could reside within the tertiary lymphoid structures which correlate with better

222 prognosis in patients with NSCLC[26]. Our findings also suggested that WDR76 expression was

223 associated with CD8 $+\mathrm{T}$ cells presence and its corresponding markers in LUAD. Despite the vital

224 role of $\mathrm{CD} 8+\mathrm{T}$ cells in antitumor immunity, an efficient antitumor immune response requires the

225 cooperation of both CD8+ and CD4+ T cells[27]. Moreover, markers of T cell exhaustion such as

226 PDCD1, LAG3 and Tim-3 were also strongly associated with WDR76 expression. Increased

227 expression of these inhibitory receptors correlated with T-cell dysfunction and disease progression

228 in NSCLC[28]. Although the effect of PD-1 blockade correlated with the amount of PD-1

229 expression, elevated LAG-3 expression was associated with insensitivity to PD-1 axis

230 blockade[29]. In addition, WDR76 expression was also positively associated with markers of

231 regulatory $\mathrm{T}$ cell (Treg) which could directly suppress the antitumor function of CD8+ T cells[30]. 
$232 \mathrm{~T}$ cell receptor (TCR) -based tracking in lung cancer revealed that many of the proliferative

233 exhausted CD8+ T cells shared the same TCR sequences (paired $\alpha$ and $\beta$ chains) with cells with

234 exhausted phenotypes[31]. The induction of the exhaustion states of these tumor-infiltrating CD8+

235 T cells could be attributable to the inhibitory ligands and suppressive immune cells[31].

236

237 There were notable limitations in our study. First, the information on relative protein levels or 238 downstream pathways involving WDR76 were unable to be provided because of only the RNA 239 sequencing data obtained from the TCGA database. Second, pathologic stage and M stage were 240 not independent prognostic factors, which might be caused by data heterogeneity. Finally, in

241 TIMER and GEPIA databases, the correlation analysis between CD8+ $\mathrm{T}$ cell infiltration and tumor

242 progression was unavailable. It was not clear from the analysis if the CD8+ T cell infiltration was 243 the effect of tumor progression or WDR76 expression, although some studies revealed no 244 significant association between CD8+ T cell density and pathologic stage in NSCLC[32, 33]. Our 245 results need to be validated by further researches.

\section{Conclusion}

Our preliminary findings showed that WDR76 was upregulated in LUAD, and high WDR76 expression was associated with clinical progression and considered as an independent risk factor for poor prognosis. WDR76 was also associated with the regulation of immune infiltrates in LUAD. Although WDR76 expression correlated with CD8+ T cells presence, it was positively 
prognostic marker for LUAD.

254

255 Competing Interests

256 The authors declare that they have no competing interests.

257 Funding

258 No funding.

\section{Author Contributions}

260

261

262

263

264

265

266

267

268

269

270

271

272

273

274

275

276

277

278

279

Drs. Likui Fang and Bo Ye contributed to the conception and design of the work. Drs. Likui Fang and Guocan $\mathrm{Yu}$ contributed to data acquisition and editing the manuscript. Drs. Wenfeng Yu and Gang Chen contributed to statistical analysis and interpretation of the data. Drs. Likui Fang and Bo Ye contributed to the revision of the manuscript. All authors have approved the final draft of the manuscript.

\section{References}

[1] Siegel RL, Miller KD, Jemal A. Cancer statistics, 2020. CA Cancer J Clin. 2020;70(1):7-30.

[2] Bray F, Ferlay J, Soerjomataram I, Siegel RL, Torre LA, Jemal A. Global cancer statistics 2018: GLOBOCAN estimates of incidence and mortality worldwide for 36 cancers in 185 countries. CA Cancer J Clin. 2018;68(6):394424.

[3] Travis WD, Brambilla E, Burke AP, Marx A, Nicholson AG. Introduction to The 2015 World Health Organization Classification of Tumors of the Lung, Pleura, Thymus, and Heart. J Thorac Oncol. 2015;10(9):1240-2. [4] Alexander M, Kim SY, Cheng H. Update 2020: Management of Non-Small Cell Lung Cancer. Lung. 2020;198(6):897-907.

[5] Piper-Vallillo AJ, Sequist LV, Piotrowska Z. Emerging Treatment Paradigms for EGFR-Mutant Lung Cancers Progressing on Osimertinib: A Review. Journal of clinical oncology : official journal of the American Society of Clinical Oncology. 2020;38(25):2926-+.

[6] Planchard D. Adjuvant Osimertinib in EGFR-Mutated Non-Small-Cell Lung Cancer. The New England journal of medicine. 2020;383(18):1780-2. 
280 [7] Yang CY, Yang JC, Yang PC. Precision Management of Advanced Non-Small Cell Lung Cancer. Annu Rev 281 Med. 2020;71:117-36.

282 [8] Mou Y, Wang J, Wu J, He D, Zhang C, Duan C, Li B. Ferroptosis, a new form of cell death: opportunities and challenges in cancer. Journal of hematology \& oncology. 2019;12(1):34.

284 [9] Chen X, Kang R, Kroemer G, Tang D. Broadening horizons: the role of ferroptosis in cancer. Nature reviews 285 Clinical oncology. 2021;18(5):280-96.

286 [10] Wu Y, Yu C, Luo M, Cen C, Qiu J, Zhang S, Hu K. Ferroptosis in Cancer Treatment: Another Way to Rome. 287 Frontiers in oncology. 2020;10:571127.

288 [11] Li Y, Yan H, Xu X, Liu H, Wu C, Zhao L. Erastin/sorafenib induces cisplatin-resistant non-small cell lung cancer cell ferroptosis through inhibition of the Nrf2/xCT pathway. Oncology letters. 2020;19(1):323-33. [12] Lou JS, Zhao LP, Huang ZH, Chen XY, Xu JT, Tai WC, Tsim KWK, Chen YT, Xie T. Ginkgetin derived from Ginkgo biloba leaves enhances the therapeutic effect of cisplatin via ferroptosis-mediated disruption of the Nrf2/HO-1 axis in EGFR wild-type non-small-cell lung cancer. Phytomedicine : international journal of phytotherapy and phytopharmacology. 2021;80:153370.

[13] Yang J, Wang F, Chen B. The role of WDR76 in diverse interactions and human diseases. Bosn J Basic Med Sci. 2021.

[14] Jiang Y, Mao C, Yang R, Yan B, Shi Y, Liu X, Lai W, Liu Y, Wang X, Xiao D, Zhou H, Cheng Y, Yu F, Cao Y, Liu S, Yan Q, Tao Y. EGLN1/c-Myc Induced Lymphoid-Specific Helicase Inhibits Ferroptosis through Lipid Metabolic Gene Expression Changes. Theranostics. 2017;7(13):3293-305.

[15] Li T, Fu J, Zeng Z, Cohen D, Li J, Chen Q, Li B, Liu XS. TIMER2.0 for analysis of tumor-infiltrating immune cells. Nucleic acids research. 2020;48(W1):W509-W14.

[16] Tang Z, Li C, Kang B, Gao G, Li C, Zhang Z. GEPIA: a web server for cancer and normal gene expression profiling and interactive analyses. Nucleic acids research. 2017;45(W1):W98-W102.

[17] Szklarczyk D, Gable AL, Nastou KC, Lyon D, Kirsch R, Pyysalo S, Doncheva NT, Legeay M, Fang T, Bork P, Jensen LJ, von Mering C. The STRING database in 2021: customizable protein-protein networks, and functional characterization of user-uploaded gene/measurement sets. Nucleic acids research. 2021;49(D1):D605-D12. WDR76 Protein Interactome. Journal of proteome research. 2019;18(9):3479-91. F, Eberl HC, Mensinga A, Brinkman AB, Lephikov K, Muller U, Walter J, Boelens R, van Ingen H, Leonhardt H, Carell T, Vermeulen M. Dynamic readers for 5-(hydroxy)methylcytosine and its oxidized derivatives. Cell. 2013;152(5):1146-59.

312 [20] Uhlen M, Zhang C, Lee S, Sjostedt E, Fagerberg L, Bidkhori G, Benfeitas R, Arif M, Liu Z, Edfors F, Sanli K, von Feilitzen K, Oksvold P, Lundberg E, Hober S, Nilsson P, Mattsson J, Schwenk JM, Brunnstrom H, Glimelius B,

314 Sjoblom T, Edqvist PH, Djureinovic D, Micke P, Lindskog C, Mardinoglu A, Ponten F. A pathology atlas of the 315 human cancer transcriptome. Science (New York, NY). 2017;357(6352): 660-+.

316 [21] Liu Z, Yanagisawa K, Griesing S, Iwai M, Kano K, Hotta N, Kajino T, Suzuki M, Takahashi T. TTF-1/NKX23171 binds to DDB1 and confers replication stress resistance to lung adenocarcinomas. Oncogene. 2017;36(26):3740-8.

318 [22] Wu Y, Zhang S, Gong X, Tam S, Xiao D, Liu S, Tao Y. The epigenetic regulators and metabolic changes in 319 ferroptosis-associated cancer progression. Molecular cancer. 2020;19(1):39.

320 [23] Huang D, Li Q, Sun X, Sun X, Tang Y, Qu Y, Liu D, Yu T, Li G, Tong T, Zhang Y. CRL4(DCAF8) dependent 

(s)

opposing stability control over the chromatin remodeler LSH orchestrates epigenetic dynamics in ferroptosis. Cell Death Differ. 2020;28(5):1593-609.

[24] Berghmans T, Dingemans AM, Hendriks LEL, Cadranel J. Immunotherapy for nonsmall cell lung cancer: a new therapeutic algorithm. The European respiratory journal. 2020;55(2):1901907.

[25] Wang W, Green M, Choi JE, Gijon M, Kennedy PD, Johnson JK, Liao P, Lang X, Kryczek I, Sell A, Xia H, Zhou J, Li G, Li J, Li W, Wei S, Vatan L, Zhang H, Szeliga W, Gu W, Liu R, Lawrence TS, Lamb C, Tanno Y, Cieslik M, Stone E, Georgiou G, Chan TA, Chinnaiyan A, Zou W. CD8(+) T cells regulate tumour ferroptosis during cancer immunotherapy. Nature. 2019;569(7755):270-4.

[26] Germain C, Gnjatic S, Tamzalit F, Knockaert S, Remark R, Goc J, Lepelley A, Becht E, Katsahian S, Bizouard G, Validire P, Damotte D, Alifano M, Magdeleinat P, Cremer I, Teillaud JL, Fridman WH, Sautes-Fridman C, DieuNosjean MC. Presence of B cells in tertiary lymphoid structures is associated with a protective immunity in patients with lung cancer. American journal of respiratory and critical care medicine. 2014;189(7):832-44.

[27] Altorki NK, Markowitz GJ, Gao D, Port JL, Saxena A, Stiles B, McGraw T, Mittal V. The lung microenvironment: an important regulator of tumour growth and metastasis. Nature reviews Cancer. 2019;19(1):9-31. [28] Thommen DS, Schreiner J, Muller P, Herzig P, Roller A, Belousov A, Umana P, Pisa P, Klein C, Bacac M, Fischer OS, Moersig W, Savic Prince S, Levitsky V, Karanikas V, Lardinois D, Zippelius A. Progression of Lung Cancer Is Associated with Increased Dysfunction of $\mathrm{T}$ Cells Defined by Coexpression of Multiple Inhibitory Receptors. Cancer immunology research. 2015;3(12):1344-55.

[29] Datar I, Sanmamed MF, Wang J, Henick BS, Choi J, Badri T, Dong W, Mani N, Toki M, Mejias LD, Lozano MD, Perez-Gracia JL, Velcheti V, Hellmann MD, Gainor JF, McEachern K, Jenkins D, Syrigos K, Politi K, Gettinger S, Rimm DL, Herbst RS, Melero I, Chen L, Schalper KA. Expression Analysis and Significance of PD-1, LAG-3, and TIM-3 in Human Non-Small Cell Lung Cancer Using Spatially Resolved and Multiparametric Single-Cell Analysis. Clinical cancer research : an official journal of the American Association for Cancer Research. 2019;25(15):4663-73. [30] Ganesan AP, Johansson M, Ruffell B, Yagui-Beltran A, Lau J, Jablons DM, Coussens LM. Tumor-infiltrating regulatory $\mathrm{T}$ cells inhibit endogenous cytotoxic $\mathrm{T}$ cell responses to lung adenocarcinoma. Journal of immunology (Baltimore, Md : 1950). 2013;191(4):2009-17.

[31] Ren X, Zhang L, Zhang Y, Li Z, Siemers N, Zhang Z. Insights Gained from Single-Cell Analysis of Immune Cells in the Tumor Microenvironment. Annu Rev Immunol. 2021;39:583-609.

[32] Donnem T, Hald SM, Paulsen EE, Richardsen E, Al-Saad S, Kilvaer TK, Brustugun OT, Helland A, LundIversen M, Poehl M, Olsen KE, Ditzel HJ, Hansen O, Al-Shibli K, Kiselev Y, Sandanger TM, Andersen S, Pezzella F, Bremnes RM, Busund LT. Stromal CD8+ T-cell Density-A Promising Supplement to TNM Staging in Non-Small Cell Lung Cancer. Clinical cancer research : an official journal of the American Association for Cancer Research. 2015;21(11):2635-43.

[33] Schulze AB, Evers G, Gorlich D, Mohr M, Marra A, Hillejan L, Rehkamper J, Schmidt LH, Heitkotter B. Tumor infiltrating $\mathrm{T}$ cells influence prognosis in stage I-III non-small cell lung cancer. Journal of thoracic disease. 2020;12(5):1824-42.

\section{Figure legends}


360

361

362

363

364

365

366

367

368

369

370

371

372

373

374

375

376

377

378

379

380

Figure 1. WDR76 expression status in LUAD. A WDR76 expression was higher in LUAD tissues than in normal tissues. B There was no statistical difference between WDR76 expression and age. C WDR76 expression was higher in male than in female. D There was no statistical difference between WDR76 expression and smoking history. E WDR76 expression was higher in T2 stage than in T1 stage, but the difference was not observed in T3 and T4 stage. F-H High WDR76 expression was associated with advanced $\mathrm{N}$ stage, $\mathrm{M}$ stage and pathologic stage. ns, $\mathrm{P} \geq 0.05$; *, $\mathrm{P}<0.05 ; * *, \mathrm{P}<0.01 ; * * *, \mathrm{P}<0.001$

Figure 2. Validation of higher WDR76 expression in LUAD than that in normal tissues in $\mathbf{A}$ GSE140797, B GSE27262 and C GSE18842 datasets. *, P<0.05; **, $\mathrm{P}<0.01$; ***, $\mathrm{P}<0.001$.

Figure 3. Kaplan-Meier survival curves for A overall survival (OS), B disease specific survival (DSS) and $\mathbf{C}$ progress free survival (PFS) of the LUAD patients with high and low WDR76 expression level.

Figure 4. Cox regression analysis of WDR76 expression and clinicopathologic characteristics with survival outcomes in LUAD. A Univariate Cox regression analysis in overall survival (OS). B Multivariate Cox regression analysis in OS. C Univariate Cox regression analysis in disease specific survival (DSS). D Multivariate Cox regression analysis in DSS. E Univariate Cox regression analysis in progress free survival (PFS). F Multivariate Cox regression analysis in PFS. 
381 Figure 5. The protein interaction network of WDR76.

382

383 Figure 6. Correlation of WDR76 expression with immune infiltration in LUAD from the TIMER

384 database. 
Figure 1

Figure 1. WDR76 expression status in LUAD.

A WDR76 expression was higher in LUAD tissues than in normal tissues. B There was no statistical difference between WDR76 expression and age. C WDR76 expression was higher in male than in female. D There was no statistical difference between WDR76 expression and smoking history. E WDR76 expression was higher in T2 stage than in T1 stage, but the difference was not observed in T3 and T4 stage. F-H High WDR76 expression was associated with advanced $\mathrm{N}$ stage, $\mathrm{M}$ stage and pathologic stage. $\mathrm{ns}, \mathrm{P} \geq 0.05 ; *, \mathrm{P}<0.05 ; * *, \mathrm{P}<0.01 ; * * *$, $\mathrm{P}<0.001$.
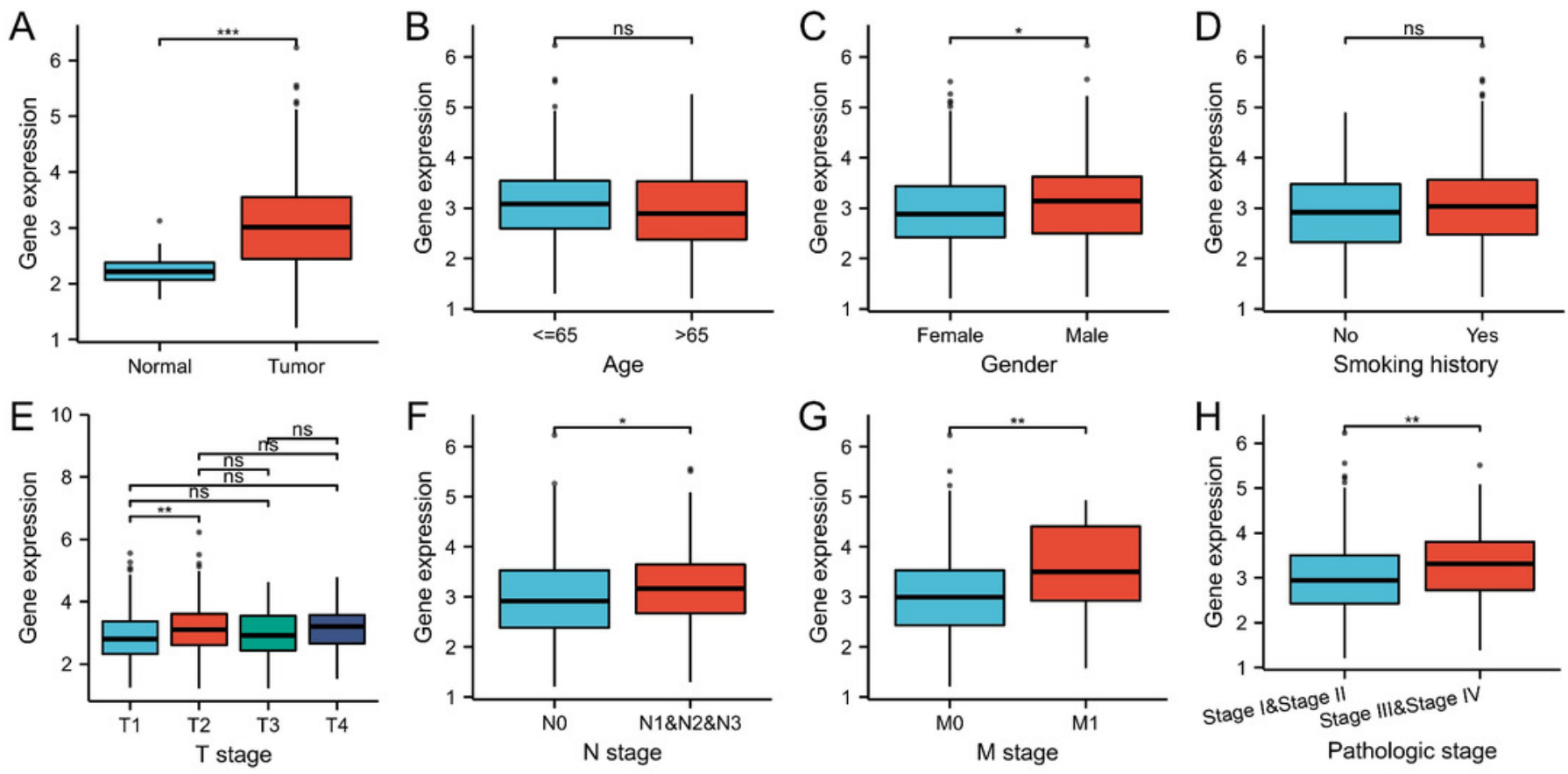
Figure 2

Figure 2. Validation of higher WDR76 expression in LUAD than that in normal tissues in A GSE140797, B GSE27262 and C GSE18842 datasets. *, P<0.05; **, P<0.01; ***, $P<0.001$.

A

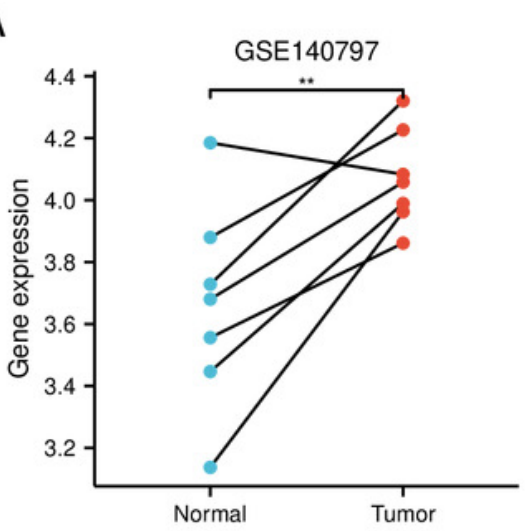

B

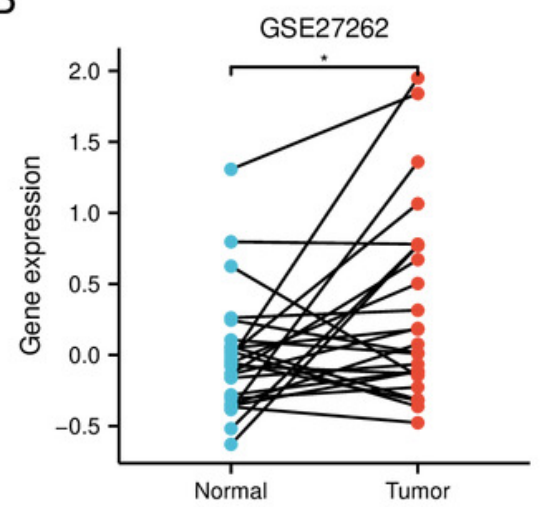

C

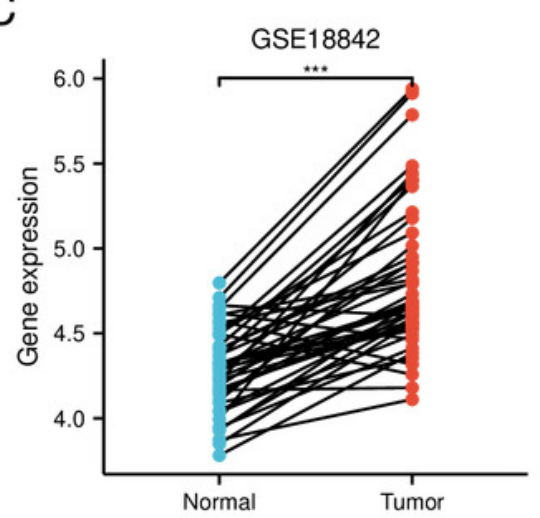


Figure 3

Figure 3. Kaplan-Meier survival curves for A overall survival (OS), B disease specific survival (DSS) and C progress free survival (PFS) of the LUAD patients with high and low WDR76 expression level.
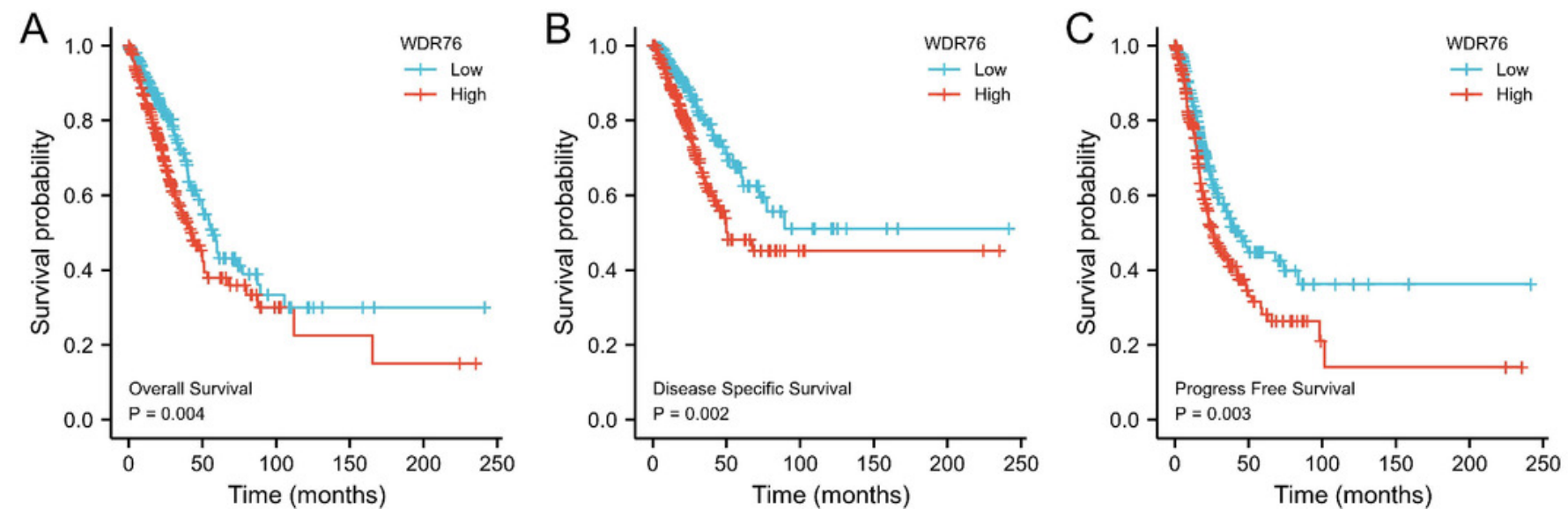


\section{Figure 4}

Figure 4. Cox regression analysis of WDR76 expression and clinicopathologic characteristics with survival outcomes in LUAD.

A Univariate Cox regression analysis in overall survival (OS). B Multivariate Cox regression analysis in OS. C Univariate Cox regression analysis in disease specific survival (DSS). D Multivariate Cox regression analysis in DSS. E Univariate Cox regression analysis in progress free survival (PFS). F Multivariate Cox regression analysis in PFS. 


\begin{tabular}{|c|c|c|c|}
\hline \multicolumn{4}{|c|}{$\begin{array}{c}\text { Univariate analysis } \\
\text { OS }\end{array}$} \\
\hline Characteristics & $\mathrm{HR}(95 \% \mathrm{Cl})$ & & $P$ value \\
\hline T stage & $1.728(1.229-2.431)$ & : & 0.002 \\
\hline $\mathrm{N}$ stage & $2.601(1.944-3.480)$ & $1 \longmapsto$ & $<0.001$ \\
\hline M stage & $2.136(1.248-3.653)$ & $\vdots \longmapsto$ & 0.006 \\
\hline Pathologic stage & $2.933(2.173-3.958)$ & $\longmapsto$ & $<0.001$ \\
\hline Gender & $1.070(0.803-1.426)$ & ter & 0.642 \\
\hline Age & $1.223(0.916-1.635)$ & $\stackrel{5}{1}$ & 0.172 \\
\hline Smoker & $0.894(0.592-1.348)$ & rân & 0.591 \\
\hline WDR76 & $1.532(1.146-2.048)$ & Iே૯ & 0.004 \\
\hline
\end{tabular}

C

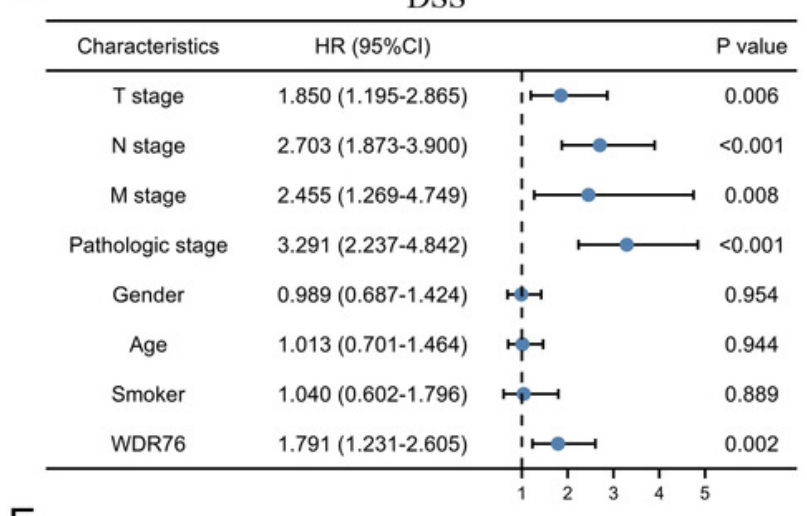

E

\begin{tabular}{|c|c|c|c|}
\hline Characteristics & $\mathrm{HR}(95 \% \mathrm{Cl})$ & & $P$ value \\
\hline T stage & $1.882(1.379-2.570)$ & $1 \longmapsto$ & $<0.001$ \\
\hline $\mathrm{N}$ stage & $1.512(1.152-1.984)$ & $: \longmapsto$ & 0.003 \\
\hline M stage & $1.513(0.855-2.676)$ & $\frac{1}{1}$ & 0.155 \\
\hline Pathologic stage & $1.960(1.502-2.557)$ & $\begin{array}{l}i \\
i\end{array}$ & $<0.001$ \\
\hline Gender & $1.172(0.901-1.526)$ & $\stackrel{\leftrightarrow}{1}$ & 0.236 \\
\hline Age & $1.023(0.784-1.335)$ & $\mapsto r-r$ & 0.867 \\
\hline Smoker & $0.968(0.658-1.426)$ & 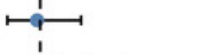 & 0.870 \\
\hline WDR76 & $1.504(1.154-1.960)$ & $i \longmapsto$ & 0.003 \\
\hline
\end{tabular}

B

Multivariate analysis

\begin{tabular}{|c|c|c|c|}
\hline Characteristics & $\mathrm{HR}(95 \% \mathrm{Cl})$ & & $P$ value \\
\hline T stage & $1.720(1.065-2.779)$ & $\stackrel{\longrightarrow}{1}$ & 0.027 \\
\hline $\mathrm{N}$ stage & $1.849(1.012-3.379)$ & $\longmapsto$ & 0.046 \\
\hline M stage & $1.507(0.803-2.830)$ & $\stackrel{1}{1} \longrightarrow$ & 0.201 \\
\hline Pathologic stage & $1.305(0.688-2.478)$ & $\stackrel{1}{\mathfrak{1}_{1}}$ & 0.415 \\
\hline Gender & $0.869(0.607-1.244)$ & $\mapsto_{1}^{-4}$ & 0.443 \\
\hline Age & $1.321(0.928-1.881)$ & $\stackrel{1}{1} \longrightarrow$ & 0.123 \\
\hline Smoker & $1.185(0.709-1.981)$ & $\stackrel{1}{n_{1}} \longrightarrow$ & 0.518 \\
\hline WDR76 & $1.468(1.031-2.089)$ & $\longmapsto$ & 0.033 \\
\hline
\end{tabular}

D

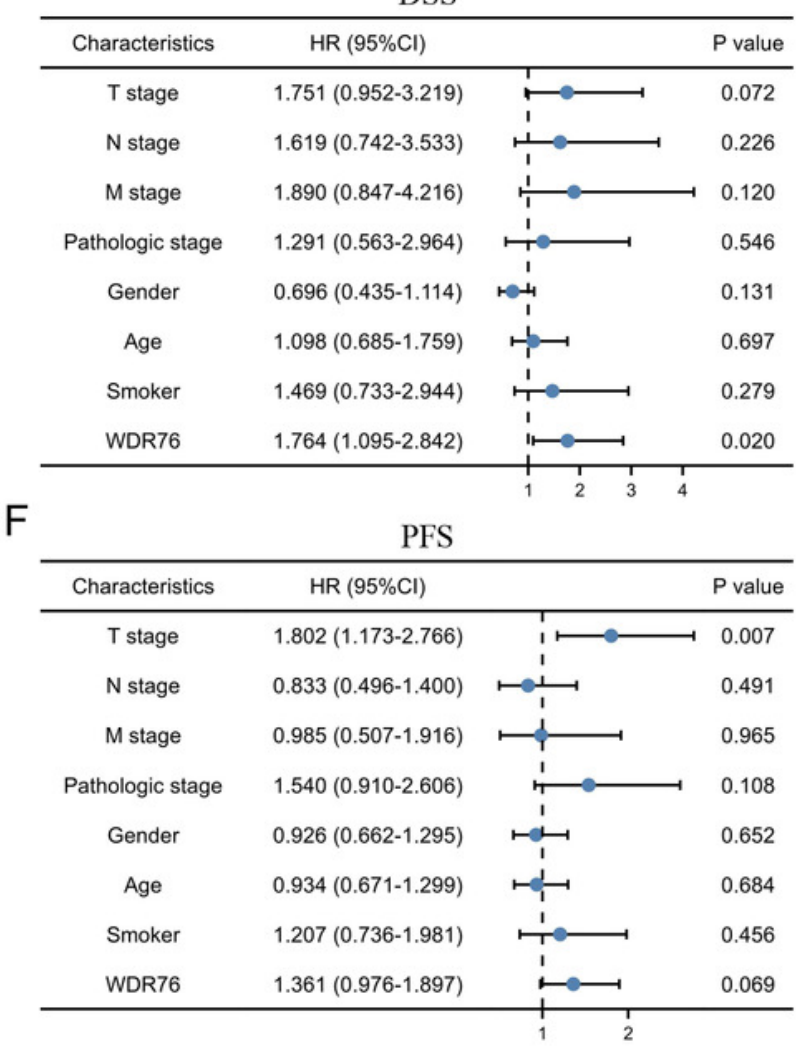


Figure 5

Figure 5. The protein interaction network of WDR76.

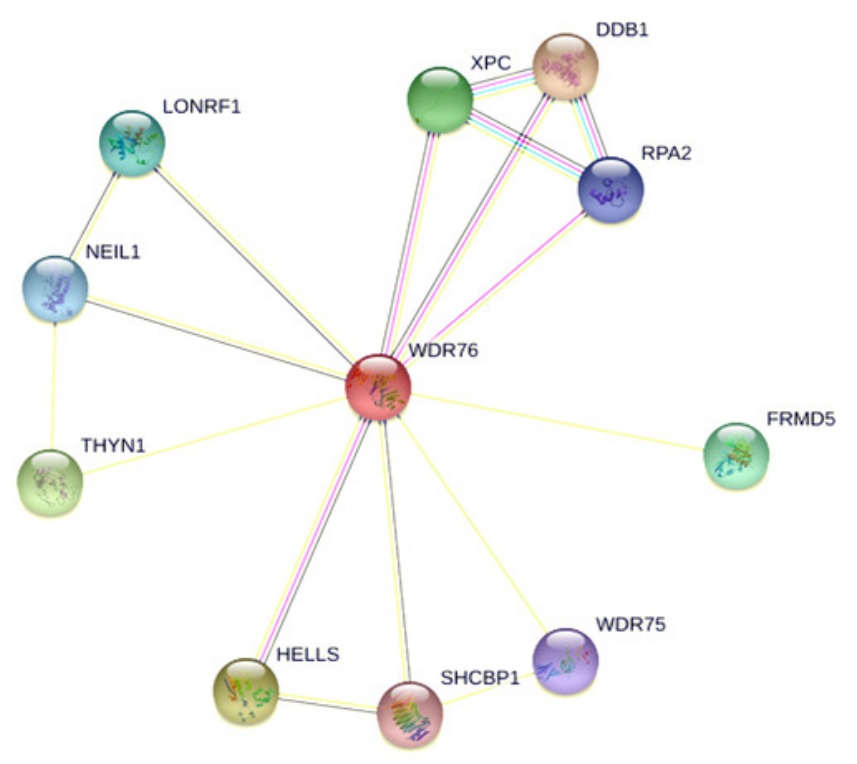

\begin{tabular}{lll}
\hline Gene symbol & Annotation & Score \\
\hline DDB1 & DNA damage-binding protein I & 0.887 \\
HELLS & Lymphoid-specific helicase & 0.824 \\
THYN1 & Thymocyte nuclear protein I & 0.749 \\
XPC & DNA repair protein complementing XP-C cells & 0.743 \\
FRMD5 & FERM domain-containing protein 5 & 0.681 \\
LONRF1 & LON peptidase N-terminal domain and ring finger 1 & 0.655 \\
NEIL1 & Endonuclease 8-like 1 & 0.643 \\
RPA2 & Replication protein A 32 kDa subunit & 0.639 \\
WDR75 & WD repeat-containing protein 75 & 0.633 \\
SHCBP1 & SHC SH2 domain-binding protein 1 & 0.629 \\
\hline
\end{tabular}


Figure 6

Figure 6. Correlation of WDR76 expression with immune infiltration in LUAD from the TIMER database.

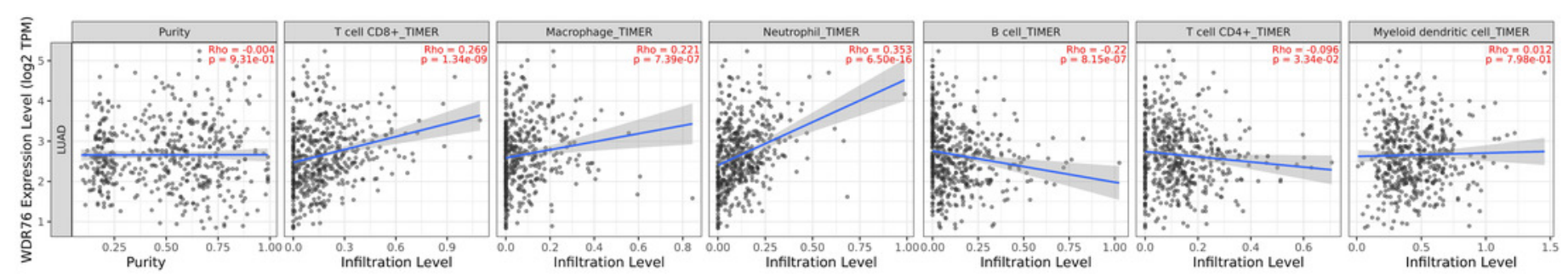




\section{Table 1 (on next page)}

Table 1. Baseline characteristics of the LUAD patients 
1 Table 1. Baseline characteristics of the LUAD patients

\begin{tabular}{llcc}
\hline Characteristic & & $\mathrm{N}$ & $(\%)$ \\
\hline Age & $\leq 65$ & 255 & 49.4 \\
\multirow{3}{*}{ Gender } & $>65$ & 261 & 50.6 \\
& Female & 286 & 53.5 \\
Smoking history & Male & 249 & 46.5 \\
& No & 75 & 14.4 \\
T stage & Yes & 446 & 85.6 \\
& T1 & 175 & 32.9 \\
& T2 & 289 & 54.3 \\
N stage & T3 & 49 & 9.2 \\
& T4 & 19 & 3.6 \\
& N0 & 348 & 67.1 \\
Pathologic stage & N1 & 95 & 18.3 \\
& N2 & 74 & 14.3 \\
M stage & N3 & 2 & 0.4 \\
& M0 & 361 & 93.5 \\
& M1 & 25 & 6.5 \\
& Stage I & 294 & 55.8 \\
& Stage II & 123 & 23.3 \\
& Stage IV & 84 & 15.9 \\
& & 26 & 4.9 \\
\hline
\end{tabular}

2 The definitions of T, N, M are primary tumor, regional lymph nodes and distant metastasis, respectively. 
Table 2 (on next page)

Table 2. Correlation analysis between WDR76 and markers of immune cells in GEPIA 
1 Table 2. Correlation analysis between WDR76 and markers of immune cells in GEPIA

\begin{tabular}{|c|c|c|c|c|c|}
\hline \multirow[t]{2}{*}{ Cell type } & \multirow[t]{2}{*}{ Gene marker } & \multicolumn{2}{|c|}{ Normal } & \multicolumn{2}{|c|}{ Tumor } \\
\hline & & $\mathrm{R}$ & Adj. P & $\mathrm{R}$ & Adj. P \\
\hline \multirow[t]{3}{*}{ B cell } & CD19 & 0.14 & 0.9 & -0.062 & 0.51 \\
\hline & KRT20 & 0.24 & 0.2 & 0.041 & 1 \\
\hline & CD38 & 0.23 & 0.25 & 0.064 & 0.48 \\
\hline \multirow[t]{2}{*}{$\mathrm{CD} 8+\mathrm{T}$ cell } & CD8A & 0.21 & 0.24 & 0.2 & $1.7 \mathrm{e}-05$ \\
\hline & CD8B & 0.11 & 0.84 & 0.21 & $4.8 \mathrm{e}-06$ \\
\hline \multirow[t]{3}{*}{ Tfh } & BCL6 & 0.25 & 0.16 & -0.0041 & 1 \\
\hline & ICOS & -0.076 & 1 & 0.12 & 0.033 \\
\hline & CXCR5 & -0.091 & 1 & -0.12 & 0.033 \\
\hline \multirow[t]{7}{*}{ Th1 } & TBX21 & 0.42 & 0.006 & 0.098 & 0.22 \\
\hline & STAT4 & 0.39 & 0.016 & 0.096 & 0.24 \\
\hline & IL12RB2 & 0.21 & 0.7 & 0.4 & $2.3 \mathrm{e}-19$ \\
\hline & IL27RA & 0.26 & 0.32 & 0.012 & 1 \\
\hline & STAT1 & 0.45 & 0.0024 & 0.48 & $1.5 \mathrm{e}-28$ \\
\hline & IFNG & 0.22 & 0.62 & 0.3 & $6 e-11$ \\
\hline & $\mathrm{TNF}$ & 0.15 & 1 & 0.02 & 1 \\
\hline \multirow[t]{4}{*}{ Th2 } & GATA3 & 0.36 & 0.021 & 0.16 & 0.002 \\
\hline & CCR3 & 0.24 & 0.27 & -0.042 & 1 \\
\hline & STAT6 & 0.38 & 0.012 & -0.11 & 0.072 \\
\hline & STAT5A & 0.32 & 0.048 & 0.077 & 0.36 \\
\hline \multirow[t]{3}{*}{ Th9 } & TGFBR2 & 0.38 & 0.009 & -0.098 & 0.096 \\
\hline & IRF4 & 0.0081 & 1 & -0.013 & 1 \\
\hline & SPI1 & -0.24 & 0.2 & -0.00019 & 1 \\
\hline \multirow[t]{4}{*}{ Th17 } & STAT3 & 0.37 & 0.018 & 0.13 & 0.015 \\
\hline & IL21R & 0.34 & 0.034 & 0.13 & 0.014 \\
\hline & IL23R & 0.046 & 1 & 0.048 & 1 \\
\hline & IL17A & -0.0046 & 1 & 0.13 & 0.014 \\
\hline \multirow[t]{2}{*}{ Th22 } & CCR10 & 0.16 & 0.46 & 0.055 & 0.46 \\
\hline & AHR & 0.28 & 0.06 & 0.088 & 0.11 \\
\hline \multirow[t]{3}{*}{ Treg } & FOXP3 & 0.21 & 0.36 & 0.11 & 0.06 \\
\hline & IL2RA & 0.15 & 0.72 & 0.26 & $1.3 \mathrm{e}-08$ \\
\hline & CCR8 & 0.22 & 0.27 & 0.15 & 0.0039 \\
\hline \multirow[t]{2}{*}{ Macrophage } & CD68 & -0.21 & 0.22 & 0.14 & 0.0038 \\
\hline & $\mathrm{CD} 11 \mathrm{~b}$ & 0.13 & 0.68 & 0.00082 & 1 \\
\hline \multirow[t]{3}{*}{ M1 } & NOS2 & 0.39 & 0.0069 & 0.075 & 0.3 \\
\hline & IRF5 & 0.029 & 1 & 0.12 & 0.022 \\
\hline & PTGS2 & 0.048 & 1 & 0.14 & 0.0069 \\
\hline \multirow[t]{2}{*}{ M2 } & CD163 & -0.21 & 0.48 & 0.18 & $2.4 \mathrm{e}-04$ \\
\hline & ARG1 & 0.15 & 1 & 0.023 & 1 \\
\hline
\end{tabular}




\begin{tabular}{|c|c|c|c|c|c|}
\hline & $\mathrm{MRC} 1$ & -0.12 & 1 & -0.066 & 0.6 \\
\hline & MS4A4A & -0.27 & 0.17 & 0.068 & 0.52 \\
\hline \multirow[t]{4}{*}{ TAM } & CCL2 & 0.019 & 1 & 0.13 & 0.012 \\
\hline & CD80 & 0.19 & 0.64 & 0.1 & 0.092 \\
\hline & CD86 & -0.12 & 1 & 0.15 & 0.0048 \\
\hline & CCR5 & 0.33 & 0.039 & 0.14 & 0.0068 \\
\hline \multirow[t]{3}{*}{ Monocyte } & CD14 & 0.1 & 1 & 0.13 & 0.011 \\
\hline & FCGR3B & 0.3 & 0.07 & 0.24 & $3.3 e-07$ \\
\hline & CD115 & 0.18 & 0.54 & 0.08 & 0.23 \\
\hline \multirow[t]{3}{*}{ Neutrophil } & CD66b & -0.0049 & 1 & -0.24 & $1.5 \mathrm{e}-07$ \\
\hline & FUT4 & 0.43 & 0.0023 & 0.22 & $4.5 \mathrm{e}-06$ \\
\hline & CD11b & 0.13 & 1 & 0.00082 & 1 \\
\hline \multirow[t]{3}{*}{ Natural killer cell } & XCL1 & 0.034 & 1 & 0.19 & 0.00009 \\
\hline & CD7 & 0.15 & 0.72 & 0.17 & 0.00036 \\
\hline & KIR3DL1 & 0.19 & 0.42 & 0.086 & 0.17 \\
\hline \multirow[t]{3}{*}{ Dendritic cell } & $\mathrm{CD} 1 \mathrm{C}$ & 0.062 & 1 & -0.33 & $1.9 \mathrm{e}-13$ \\
\hline & CD141 & 0.25 & 0.18 & -0.1 & 0.075 \\
\hline & $\mathrm{CD} 11 \mathrm{c}$ & -0.031 & 1 & 0.046 & 0.93 \\
\hline \multirow[t]{4}{*}{$\mathrm{T}$ cell exhaustion } & PDCD1 & 0.28 & 0.12 & 0.18 & 0.0003 \\
\hline & CTLA4 & 0.25 & 0.22 & 0.11 & 0.076 \\
\hline & LAG3 & 0.4 & 0.0068 & 0.26 & $2.5 \mathrm{e}-08$ \\
\hline & TIM-3 & -0.21 & 0.44 & 0.14 & 0.008 \\
\hline
\end{tabular}

2 Adj, adjusted; Tfh, follicular helper T cell; Th, T helper cell; Treg, regulatory $\mathrm{T}$ cell; TAM, tumor-associated 3 macrophage 